\title{
AN UNUSUAL CASE OF SHOULDER PAIN DUE TO SEPTIC ARTHRITIS OF THE STERNOCLAVICULAR JOINT IN A SYSTEMIC LUPUS ERYTHEMATOSUS PATIENT
}

Marina Barguil Macêdo ${ }^{1, *}$, Isabele Parente de Brito Antonelli ${ }^{1}$, André Silva Franco ${ }^{1}$, Jean Michell Correia Monteiro ${ }^{1}$, Osvaldo Luiz Camata Junior ${ }^{1}$, Luma Mendes Brito ${ }^{1}$, Luciana Parente Costa Seguro ${ }^{1}$, Henrique Ayres Mayrink Giardini ${ }^{1}$, Lissiane Karine Noronha Guedes ${ }^{1}$, Rosa Maria Rodrigues Pereira ${ }^{1}$

1. Universidade de São Paulo (USP), São Paulo (SP), Brazil.

*Corresponding author: marina.macedo@hc.fm.usp.br

\section{BACKGROUND}

The sternoclavicular joint (SCJ) is a rather uncommon site of septic arthritis (SA), which usually develops in patients with predisposing factors, such as intravenous drug use or diabetes mellitus. Up until now, there has been no description of SCJ SA in a systemic lupus erythematosus (SLE) patient.

\section{CASE REPORT}

A 23-year-old African Brazilian female presented with a 2-months history of incapacitating mechanical right shoulder pain. She reported a weight loss of $3 \mathrm{~kg}$ on this period, but denied fever or other constitutional symptoms. On physical examination, mild wasting of the right deltoid muscle was noticed, attributed to underuse, but shoulder examination was otherwise unremarkable. The patient had been diagnosed with SLE eight months earlier. At the time of diagnosis, she presented with nephrotic syndrome and a large pericardial effusion which required pericardiostomy. Pericardial fluid cultures for bacteria, fungi and mycobacteria were negative, and the effusion abated after starting of pulse therapy with methylprednisolone and cyclophosphamide. Four months later, she developed a soft tissue abscess on the previous site of the pericardiostomy. Culture of the abscess yielded Pseudomonas aeruginosa. She was treated with a 10-day course of cefepime, with complete resolution of the skin infection. On the current admission, she presented with a CRP of $47.2 \mathrm{mg} / \mathrm{L}$, an ESR of $134 \mathrm{~mm} / 1^{\text {st }} \mathrm{h}$, and a mild leukocytosis of $11,060 / \mathrm{mm}^{3}$, with no left shift. Shoulder MRI did not show any abnormality, neither did a chest CT. Due to these negative results, a whole body FDG-PET/ CT was requested. The PET/CT scan evidenced marked focal hypercaptation measuring $2.8 \times 2.0 \times 3.7 \mathrm{~cm}$ on the right SCJ (SUV max of 9.6), with bone erosion and central necrosis on the lateral border of the manubrium (Fig. 1). A CT-guided biopsy of the bone and aspiration of the synovial fluid yielded a $P$. aeruginosa with the same antibiotic sensitivity profile as the one cultured from the skin abscess. The patient was started on intramuscular gentamycin and discharged with a proposal to continue the treatment for 4 weeks, and later on repeat the image to confirm resolution of the infection. She evolved with substantial improvement of the pain and decrease of inflammatory markers.

\section{CONCLUSION}

A highly unusual case of SCJ SA with adjacent osteomyelitis of the sternum manifesting as shoulder pain in a young immunosuppressed SLE patient is presented. The infection was regarded as a complication of the pericardiostomy performed at the initial presentation of SLE. 


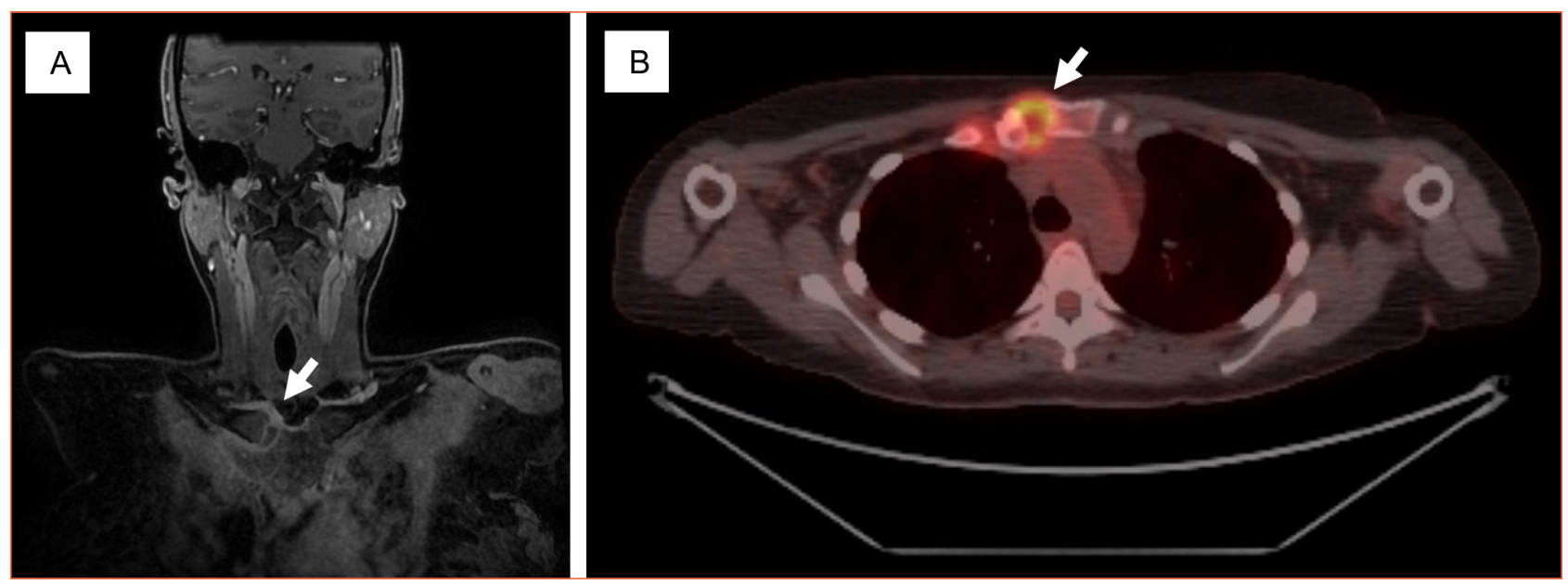

Figure 1. Evidence of an active inflammatory process on the right sternoclavicular joint by means of MRI and FDG-PET/CT Scan.

A) Coronal view of brachial plexus MRI, on gradient-echo sequence, evidencing synovitis of the right SCJ (arrow). B) Axial view of FDG-PET/CT showing a focal lesion on SCJ with marked hypercaptation (SUV max of 9.6 - arrow), and a central area suggestive of necrosis. 